\title{
3C 273 : A STAR-LIKE OBJECT WITH LARGE RED-SHIFT
}

\author{
By Dr. M. SCHMIDT \\ Mount Wilson and Palomar Observatories, Carnegie Institution of Washington, California Institute of Technology, Pasadena
}

$\mathrm{T}$ HE only objects seen on a 200-in. plate near tho positions of the components of the radio source $3 C 273$ reported by Hazard, Mackey and Shimmins in the preceding article are a star of about thirteonth magnitude and a faint wisp or jet. The jet has a width of $1^{\prime \prime}-2^{\prime \prime}$ and extends away from the star in position angle $43^{\circ}$. It is not visible within $11^{\prime \prime}$ from the star and ends abruptly at $20^{\prime \prime}$ from the star. The position of the star, kindly furnished by Dr. T. A. Matthews, is R.A. $12 \mathrm{~h} 26 \mathrm{~m} \mathrm{33} \cdot 35 \mathrm{~s} \pm 0 \cdot 04 \mathrm{~s}$, Decl. $+2^{\circ} 19^{\prime} 42 \cdot 0^{\prime \prime} \pm 0 \cdot 5^{\prime \prime}(1950)$, or $1^{\prime \prime}$ east of component $B$ of the radio source. The end of the jet is $1^{\prime \prime}$ east of component $A$. The close correlation between the radio structure and the star with the jet is suggestive and intriguing.

Spectra of the star were taken with the prime-focus spectrograph at the 200 -in. telescope with dispersions of 400 and $190 \AA$ per $\mathrm{mm}$. They show a number of broad. emission foatures on a rather blue continuum. The most prominent features, which have widths around, $50 \AA$, are, in order of strength, at $5632,3239,5792,5032 \AA$. These and other weaker emission bands are listed in the first column of Table 1. For three faint bands with widths of $100-200 \AA$ the total range of wave-length is indicated.

The only explanation found for the spectrum involves a considerable red-shift. A red-shift $\Delta \lambda / \lambda_{0}$ of 0.158 allows identification of four emission bands as Balmer lines, as indicated in Table 1. Their relative strengths are in agreement with this explanation. Other identifications based on the above red-shift involve the Mg II lines around $2798 \AA$, thus far only found in emission in the solar chromosphere, and a forbidden line of [O III] at $5007 \AA$. On this basis another [O III] line is expected at $4959 \AA$ with a strength one-third of that of the line at $5007 \AA$. Its detectability in the spectrum would be marginal. A weak emission band suspected at $5705 \AA$, or $4927 \AA$ reduced for red-shift, does not fit the wave-length. No explanation is offered for the three very wide emission bands.

It thus appears that six omission bands with widths around $50 \AA$ can be explained with a red-shift of 0.158 . The differences between the observed and the expected wave-lengths amount to $6 \AA$ at the most and can be entirely understood in terms of the uncertainty of the measured wave-lengths. The present explanation is supported by abservations of the infra-red spectrum communicated by

\begin{tabular}{|c|c|c|c|}
\hline$\lambda$ & $\lambda / 1 \cdot 158$ & $\lambda_{0}$ & \\
\hline $\begin{array}{l}3239 \\
4595 \\
4753 \\
5032\end{array}$ & $\begin{array}{l}2797 \\
3968 \\
4104 \\
4345\end{array}$ & $\begin{array}{l}2798 \\
3970 \\
4102 \\
4340\end{array}$ & $\begin{array}{l}\mathrm{Mg} \text { II } \\
\mathbf{H e} \\
\mathrm{H} \delta \\
\mathrm{H} \gamma\end{array}$ \\
\hline $\begin{array}{c}5200-5415 \\
5632 \\
5792 \\
6005-6190 \\
6400-6510\end{array}$ & $\begin{array}{c}4490-4675 \\
4864 \\
5002 \\
5186-5345 \\
5527-5622\end{array}$ & $\begin{array}{l}4861 \\
5007\end{array}$ & $\begin{array}{l}\mathbf{H} \beta \\
{[0 \text { III] }}\end{array}$ \\
\hline
\end{tabular}

Oke in a following article, and by the spectrum of another star-like object associated with the radio source $3 C 48$ discussed by Greonstein and Matthews in another communication.

The unprecedented identification of the spectrum of an apparently stellar object in terms of a large red-shift suggests either of the two following explanations.

(1) The stellar object is a star with a large gravitational red-shift. Its radius would then be of the order of $10 \mathrm{~km}$. Preliminary considerations show that it would be extremely difficult, if not impossible, to account for the occurrence of permitted lines and a forbidden line with the same redshift, and with widths of only 1 or 2 per cent of the wavelength.

(2) The stellar object is the nuclear region of a galaxy with a cosmological red-shift of $0 \cdot 158$, corresponding to an apparent velocity of $47,400 \mathrm{~km} / \mathrm{sec}$. The distance would be around 500 megaparsecs, and the diameter of the nuclear region would have to be less than 1 kiloparsec. This nuclear region would be about 100 times brighter optically than the luminous galaxies which have been identified with radio sources thus far. If the optical jet and component $\boldsymbol{A}$ of the radio source are associated with the galaxy, they would be at a distanee of 50 kiloparsecs, implying a time-scale in excess of $10^{5}$ years. The total energy radiated in the optical range at constant luminosity would be of the order of $10^{59} \mathrm{ergs}$.

Only the detection of an irrefutable proper motion or parallax would definitively establish $3 C 273$ as an object within our Galaxy. At the present time, however, the explanation in terms of an extragalactic origin seems most direct and least objectionable.

I thank Dr. T. A. Matthews, who directed my attention to the radio source, and Drs. Greenstein and Oke for valuable discussions.

\section{ABSOLUTE ENERGY DISTRIBUTION IN THE OPTICAL SPECTRUM OF 3C 273}

\author{
By DR. J. B. OKE
}

Mount Wilson and Palomar Observatories, Carnegie Institution of Washington, California Institute of Technology, Pasadena

$\mathrm{T}$ HE radio source $3 C 273$ has recently beon identified with a thirteenth magnitude star-like object. The details are given by $M$. Schmidt in the preceding communication. Since $3 C 273$ is relatively bright, photoelectric spectrophotometric observations were mado with the 100-in. telescope at Mount Wilson to determine the absolute distribution of energy in the optical region of the spectrum; such observations are useful for determining if synchrotron radiation is present. In the wave-length region between $3300 \AA$ and $6000 \AA$ measurements were made in 16 selected $50-\AA$ bands. Continuous spectral scans with a resolution of $50 \AA$ were also made. The measurements were placed on an absolute-energy system by also observing standard stars whose absolute enorgy distributions were known ${ }^{1}$. The accuracy of the 16 selected points is approximately 2 per cent. The strong emission features found by Schmidt were readily detected; other very faint features not apparent on Schmidt's spectra may be present.

The source $3 C 273$ is considerably bluer than the other known star-like objects $3 C 48,3 C$ 196, and $3 C 286$ which have been studied in detail ${ }^{2}$. The absolute energy distribution of the apparent continuum can be accurately represented by the equation:

$$
F_{v} \propto v^{+0.28}
$$

where $F_{y}$ is the flux per unit frequency interval and $v$ is the frequency. The apparent visual magnitude of $3 C 273$ is $+12 \cdot 6$, which corresponds to an absolute flux at the Earth of $3.5 \times 10^{-28} \mathrm{~W} \mathrm{~m} \mathrm{~m}^{-2}(\mathrm{c} / \mathrm{s})^{-1}$ at 5600 A. At 